\title{
Entrenamiento de resistencia muscular en jóvenes universitarios ¿método de oclusión vascular o tradicional?
}

\author{
Resistance training in college students. \\ Vascular occlusion training or traditional method? \\ *José Bimbela-Villalobos, *Marisela Suárez-García, **Jorge Alberto Aburto-Corona, \\ **Luis Mario Gómez-Miranda
}

Bimbela-Villalobos, J., Suárez-García, M., Aburto-Corona, J., Gómez-Miranda, L. (2018). Entrenamiento de resistencia muscular en jóvenes universitarios ¿método de oclusión vascular o tradicional? Revista Ciencias de la Actividad Física $U C M, \mathrm{~N}^{\circ}$ 19(2), julio-diciembre, 1-8. DOI: http://doi.org/10.29035/rcaf.19.2.8

\section{RESUMEN}

El objetivo del estudio fue determinar si existen diferencias significativas en la circunferencia del brazo y en el número de repeticiones realizadas en base a dos métodos de entrenamiento: oclusión vascular y tradicional. Se reclutaron ocho sujetos a los cuales, de manera aleatoria, se les asignó el método de oclusión vascular (OVbi) para un brazo y el entrenamiento tradicional (ETmi) para el otro. Se realizó un pretest en el cual se midió la circunferencia del brazo y la cantidad de repeticiones que pudieran realizar con una carga equivalente al $9 \%$ de la masa corporal. Después de cuatro semanas de tratamiento (tres sesiones por semana) se realizó el postest. Los resultados no encontraron interacción significativa en la circunferencia del brazo $(\mathrm{p}=0.164)$ ni en la cantidad de repeticiones $(\mathrm{p}=0.390)$, sin embargo, si se encontraron diferencias en la cantidad de repeticiones realizadas entre el pretest y postest $(\mathrm{p}=0.048)$. Estos resultados demuestran que, independientemente del tratamiento (OVbi y ETmi), los sujetos aumentaron el número de repeticiones realizadas, en pocas palabras, un entrenamiento a baja intensidad con oclusión vascular durante cuatro semanas es igual de efectivo a un entrenamiento tradicional de moderada intensidad.

\section{PALABRAS CLAVE}

Restricción de flujo sanguíneo, fuerza muscular, KAATSU.

\begin{abstract}
The purpose of the study was to determine the effect of vascular occlusion (VO) and traditional resistance training (TRT) methods on arm circumference and number of repetitions to exhaustion. Eight participants were randomly assigned to VO in one arm and TRT on the other. Before and after four weeks of training performed three times per week, measurements were obtained on arm circumference and number of repetitions with a workload equivalent to $9 \%$ of body mass. No significant treatment by measurements interactions were found on arm circumference $(\mathrm{p}=0.164)$ and number of repetitions to exhaustion $(\mathrm{p}=0.390)$. Regardless of the training method, participants improved number of repetition to exhaustion following the intervention $(p=0.048)$. In conclusion, four weeks of VO is as effective as TRT for improving arm muscular performance to exhaustion.
\end{abstract}

\section{Key words}

Blood flow restriction, muscular strength, KAATSU.

\footnotetext{
* Licenciados en Actividad Física y Deporte. Facultad de Deportes, Universidad Autónoma de Baja California, México.

*** Profesores Investigadores del Cuerpo Académico UABC-CA-230 Ejercicio Físico y Salud. Facultad de Deportes, Universidad Autónoma de Baja California, México.
} 


\section{INTRODUCCIÓN}

El entrenamiento de fuerza ha sido utilizado con el propósito de mejorar esta cualidad física. Existen registros que evidencian el entrenamiento de fuerza con pesas en forma de piedra en la población china desde los años 3600 a.C., asimismo en Egipto, India y Grecia. Durante mucho tiempo se ha realizado entrenamiento de resistencia muscular con pesas, sin embargo, Archibald MacLaren fue quien se apropió del primer sistema de entrenamiento físico basado en mancuernas y barras con peso, además de formalizar el principio de entrenamiento de sobrecarga progresiva (Verkhoshansky \& Stiff, 2011).

Existen diversos métodos para el incremento de la fuerza e hipertrofia muscular (Lin \& Chen, 2012). La mayoría de estos métodos involucran ejercicios de alta intensidad, debido a que se cree que el ejercicio de resistencia con carga menor a $70 \%$ de una repetición máxima (1RM) no genera hipertrofia muscular (Dankel, Jessee, Abe \& Loenneke, 2016). Por esta razón, el entrenamiento con oclusión vascular a baja intensidad (OVbi) se ha propuesto como una alternativa al ejercicio de altas intensidades, para la rehabilitación, aumento de la fuerza y masa muscular (Garber et al., 2011).

El entrenamiento OVbi fue originalmente concebido y desarrollado en Japón a finales de la década de los 60's por Yoshiaki Sato y se denomina entrenamiento KAATSU. Antes del año 2008, los instrumentos para entrenamiento de oclusión vascular eran escasos fuera de Japón (Garber et al., 2011). Algunos investigadores mencionan que la hipertrofia causada por el entrenamiento OVbi se debe a la acumulación de metabolitos, hinchamiento de células, al mayor reclutamiento de unidades motoras, proliferación de células satélite y a la disminución de miostatina (Loenneke, et al. 2012; Loenneke, Fahs, Wilson \& Bemben, 2011; Ogasawara Loenneke, Thiebaud \& Abe, 2013; Nielsen, et al. 2012).

Uno de los componentes de la prescripción de ejercicio es la intensidad de la carga (DeWeese, Hornsby, Stone, \& Stone, 2015). En el entrenamiento con oclusión vascular, inten- sidad baja como el 20\% de 1RM, puede llegar a generar mejoras significativas en la fuerza e hipertrofia muscular (Vanwye, Weatherholt, \& Mikesky, 2017). Takarada et al. (2000), encontraron que entrenar a baja intensidad con oclusión vascular tiene mayor efecto que entrenar solo a baja intensidad, y similar a entrenar a intensidades medias-altas en actividad muscular, área transversal del musculo y en fuerza muscular, aunque el entrenamiento de oclusión vascular aumenta más la concentración de lactato.

Takarada, Sato, \& Ishii (2002), encontraron que el entrenamiento de oclusión vascular a baja intensidad mejora la fuerza, resistencia e hipertrofia muscular de jugadores profesionales de rugby. Cabe mencionar que estos jugadores se consideran adaptados, debido a que tenían una media de cinco años realizando entrenamientos de fuerza.

Takarada, Sato, \& Ishii (2004), descubrieron que al realizar extensiones de pierna, la oclusión vascular, o el entrenamiento a baja intensidad por sí solo no tenían efectos significantes, pero al aplicar ambos al mismo tiempo, se encontró que aumenta la fuerza isométrica e isocinética, el área transversal de los extensores de rodilla y la concentración de hormona de crecimiento en plasma después de la sesión de entrenamiento.

Como lo anterior, Sousa et al. (2017) encontraron que el entrenamiento de pesas a baja intensidad con oclusión vascular, a alta intensidad o combinados, producen resultados similares en activación muscular, torque y resistencia muscular local. Por esta razón, el objetivo de este estudio fue determinar si existen diferencias significativas en la circunferencia del brazo y en el número de repeticiones realizadas en base a dos métodos de entrenamiento: OVbi y el entrenamiento tradicional de moderada intensidad (ETmi).

\section{METODOLOGÍA}

Sujetos. Se reclutaron nueve varones estudiantes universitarios físicamente activos y aparentemente sanos (ACSM, 2005). Todos los 
sujetos leyeron y firmaron una carta de consentimiento informado. A todos ellos se les pidió que durante el estudio evitaran realizar trabajo físico en el tren superior, específicamente de brazos. A un sujeto le surgió una molestia en el bíceps braquial durante el tratamiento, por tal motivo se dio de baja del protocolo. Al final del estudio solo se analizaron los datos de ocho sujetos (edad $20.8 \pm 1.8$ años; estatura $177.3 \pm 7.1$ centímetros; peso $73.9 \pm 12.9$ kilogramos).

Procedimiento. Después de haber firmado la carta consentimiento se les midió la circunferencia de ambos brazos. Se evaluó la presión arterial con y sin oclusión vascular, después, se determinó el promedio en base a la fórmula mencionada por Sainas et al. (2016) (presión arterial promedio $=$ presión arterial diastólica $(\mathrm{PAD})+1 / 3$ [presión arterial sistólica - PAD]). No se encontraron diferencias significativas entre la presión arterial promedio sin $(83.9 \pm$ $6.7 \mathrm{mmHg})$ y con oclusión vascular $(83.9 \pm$ $11.3 \mathrm{mmHg})(\mathrm{p}>.05)$.

Los sujetos asistieron a doce sesiones (cuatro semanas) con un día como mínimo de reposo entre sesión. Antes de la primera sesión, se hizo una medición de circunferencia de brazo extendido-relajado (en la terminación de la inserción distal del musculo deltoides), además, se hizo una prueba de repeticiones máximas de curl de bíceps martillo utilizando el 9\% de la masa corporal (George, Fisher, \& Vehrs, 2005). Las mediciones se realizaron en ambos brazos, debido a que uno fue escogido de manera aleatoria para hacer ejercicio con oclusión vascular a baja intensidad (OVbi) y el otro para ejecutar el método de entrenamiento tradicional de moderada intensidad (ETmi). Después de realizar la prueba de repeticiones máximas, se aplicó la fórmula de Baechle, Earle y Wathen (2000), para determinar la repetición máxima (1RM). Cabe mencionar que solo un sujeto era de brazo izquierdo dominante. Durante el transcurso de la investigación, se les pidió no realizar ejercicio del tren superior, con el propósito de que no alterara los posibles efectos de la variable independiente. Aun así, los sujetos reportaron realizar solamente ejercicio aeróbico (trote).

Durante las sesiones, los sujetos realizaron el ejercicio curl de bíceps de pie, a diferentes intensidades (ver tabla 1), con una cadencia de 2-0-2-0 propuesta por el ACSM (2009). Para el brazo que entrenaba con oclusión vascular a baja intensidad se utilizó un velcro de 1.9 centímetros de ancho para disminuir un 5\% la circunferencia del brazo. El brazo que hacía entrenamiento tradicional a moderada intensidad no se le puso velcro, simplemente hizo el ejercicio como usualmente se hace. Los sujetos no tenían experiencia realizando este ejercicio.

Tabla 1

Dosificación de la carga de trabajo durante las cuatro semanas de tratamiento.

\begin{tabular}{|c|c|c|c|c|c|c|c|}
\hline Condición & & Orden & Repeticiones & $\begin{array}{c}\text { Recuperación } \\
\text { (segundos) }\end{array}$ & $\begin{array}{c}\# \\
\text { Series }\end{array}$ & $\begin{array}{c}\text { Intensidad } \\
\text { (\% 1RM) }\end{array}$ & Cadencia \\
\hline \multirow{6}{*}{ OVbi } & \multirow{3}{*}{ 1a sesión } & 1 & 30 & 30 & 1 & 20 & \multirow{3}{*}{$2-0-2-0$} \\
\hline & & 2 & 15 & 30 & 3 & 20 & \\
\hline & & 3 & 20 & - & 1 & 20 & \\
\hline & \multirow{3}{*}{$2 \mathrm{a}$ a $12 \mathrm{a}$ sesión } & 1 & 30 & 30 & 2 & 20 & \multirow{3}{*}{$2-0-2-0$} \\
\hline & & 2 & 15 & 30 & 2 & 20 & \\
\hline & & 3 & 50 & - & 1 & 20 & \\
\hline \multirow{3}{*}{ ETmi } & Calentamiento & & 10 & $<60$ & 1 & 32.5 & Al gusto \\
\hline & \multirow{2}{*}{ 1a a 12 a sesión } & 1 & 12 & 60 & 4 & 65 & \multirow{2}{*}{$2-0-2-0$} \\
\hline & & & MRP & - & 1 & 65 & \\
\hline
\end{tabular}

Nota: MRP=Máximas Repeticiones Posibles; 1RM=Repetición Máxima. 
Análisis estadístico. Se hizo estadística descriptiva para la variable edad, talla y peso. Seguido, se realizó una prueba de normalidad de datos Shapiro-Wilk, con la cual se determinó que la distribución de datos es normal ( $\mathrm{p}$ $>0.05$ ). Por tal razón, se ejecutó un ANOVA de dos vías (tratamiento por mediciones) de muestras independientes para la circunferencia en brazo y para el número de repeticiones realizadas de curl de bíceps. Para el análisis post hoc se utilizó el test de Bonferroni, tomando como significancia un valor de $\mathrm{p}<$ 0.05 .

\section{RESULTADOS}

En base a un análisis de varianza de dos vías de muestras independientes no se encontró interacción significativa en la circunferencia del brazo después de cuatro semanas de tratamiento ( $\mathrm{p}=0.164)$, de la misma manera, no se hallaron diferencias en los efectos simples de mediciones (pre $28.7 \pm 2.2 \mathrm{~cm}$ y postest $28.8 \pm$ $2.9 \mathrm{~cm} ; \mathrm{p}=0.610)$ ni tratamiento (OVbi $28.8 \pm$ $2.6 \mathrm{~cm}$ y ETmi $28.7 \pm 2.6 \mathrm{~cm} ; \mathrm{p}=0.925$ ) (figura 1). Estos resultados indican que, independientemente del entrenamiento de oclusión vascular o tradicional, la circunferencia del brazo será la misma después de 12 sesiones de entrenamiento en cuatro semanas. Cabe mencionar que el entrenamiento OVbi se realizó con el $20 \%$ de 1 RM y el de ETmi con el $65 \%$ de 1 RM.
Lo mismo sucedió con las repeticiones realizadas, en donde la interacción entre tratamiento y mediciones no fue significativa $(\mathrm{p}=$ 0.390 ), al igual que el efecto simple tratamiento (OVbi $18.7 \pm 9.1$ repeticiones y ETmi 19.1 \pm 8.2 repeticiones; $\mathrm{p}=0.628$ ). Sin embargo, se encontraron diferencias en la variable mediciones (pretest $16.9 \pm 7.8$ repeticiones y postest $20.9 \pm 9.0$ repeticiones; $\mathrm{p}=0.048$ ) (figura 2 ). Estos resultados significan que, independientemente del tratamiento (OVbi y ETmi), los sujetos aumentaron el número de repeticiones realizadas, en pocas palabras, un entrenamiento a baja intensidad con oclusión vascular es igual de efectivo a un entrenamiento tradicional de moderada intensidad.

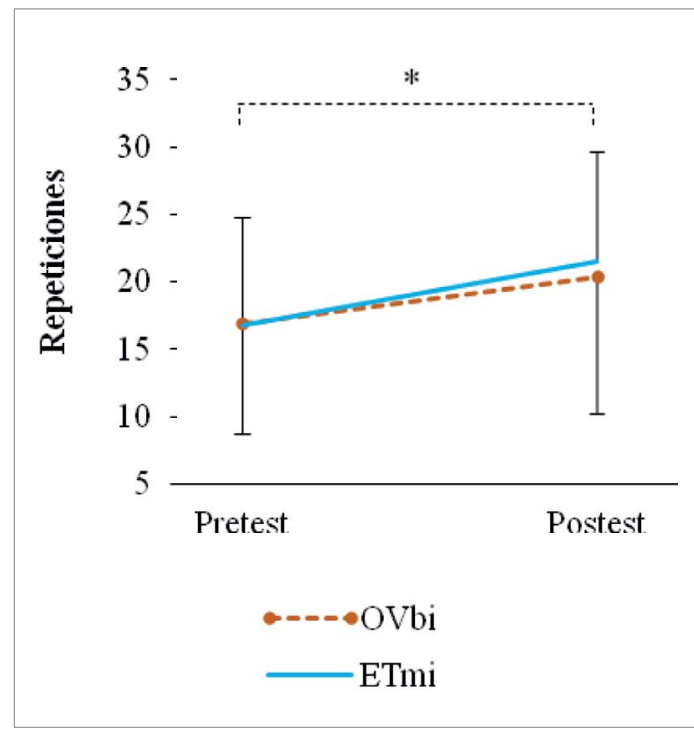

Figura 2. Comparación entre los diferentes métodos en las mediciones en las repeticiones realizadas.

${ }^{\star}$ Diferencia significativa entre pretest y postest ( $\mathrm{p}$ $=0.048$ ).

Figura 1. Comparación entre los diferentes métodos en las mediciones en la circunferencia del brazo. 


\section{DISCUSIÓN}

El hallazgo más importante de este estudio fue que el entrenamiento de OVbi (20\% 1RM) genera el mismo efecto que el ETmi (65\% 1RM), debido a que ambas condiciones mejoraron el número de repeticiones realizadas de curl de bíceps entre el pretest y postest en cuatro semanas de este mismo ejercicio. Fahs, et al. (2014), son otros investigadores que encontraron resultados similares, sin embargo, el grupo de OVbi realizaba ejercicio con el 30\% 1RM y el otro a $60 \% 1 \mathrm{RM}$, además de que evaluaron músculos del tren inferior (cuádriceps) y utilizaron sujetos de mediana edad (42 a 62 años).

En la presente investigación no se encontró diferencia en la circunferencia de brazo después de cuatro semanas de entrenamiento de curl de bíceps de pie. Esto pudo deberse a que no se tomó en cuenta la composición corporal para determinar los porcentajes de grasa y músculo en ambos brazos, es decir, los sujetos pudieron disminuir la grasa, aumentado el músculo y al finalizar obtener la misma circunferencia de brazo.

Un estudio muy similar a este es el de Lixandrão, et al. (2015), donde combinaron intensidades de ejercicio y de oclusión vascular (OV) en cinco grupos distintos: OV-20/40, OV-20/80, OV-40/40, OV-40/80 y un grupo sin oclusión vascular que trabajaba al $80 \%$ (OV/80) (porcentaje de 1RM/porcentaje de restricción del flujo sanguíneo, respectivamente). Encontraron que 12 semanas de entrenamiento pueden generar un aumento de hasta $5 \%$ en la masa muscular de los cuádriceps (OV-40/80 y OV/80). Cabe mencionar que los investigadores realizaron resonancia magnética del plano axial para determinar la proporción de los músculos cuádriceps. Otros investigadores concuerdan con estos hallazgos (Lowery, et al., 2013; Vechin, et al., 2015).

Lowery, et al. (2013), realizaron un estudio con el objetivo de determinar si ocho semanas de ejercicio de resistencia aumenta el grosor muscular del bíceps braquial. Para llevar a cabo el proyecto, un brazo realizaba entrenamiento de resistencia a baja intensidad ( $30 \%$ 1RM) con oclusión vascular $(\mathrm{OV})$ mientras que el otro brazo lo hacía a moderada intensidad (60\% 1RM) sin OV. Se realizó una medición del grosor del bíceps al inicio, otra a las cuatro semanas y la otra al finalizar el protocolo utilizando ultrasonido directo. Los investigadores concluyen que el entrenamiento de oclusión vascular a baja intensidad (30\% 1RM) estimula el músculo de la misma manera que el entrenamiento a moderada intensidad sin oclusión vascular (60\% 1RM).

Una investigación similar, con entrenamiento concurrente en lugar del entrenamiento de pesas, es la de Libardi, et al. (2014). En este estudio, los investigadores reclutaron a 25 sujetos, los cuales fueron evaluados antes y después de doce semanas en el área muscular de cuádriceps, $1 \mathrm{RM}$ y $\mathrm{VO}_{2}$ máx. Divididos en tres grupos: entrenamiento concurrente (50$80 \%$ del $\mathrm{VO}_{2}$ máx), oclusión vascular a baja intensidad (20-30\% 1RM) y un grupo control. Después de doce semanas de tratamiento, encontraron un incremento similar en el área de cuádriceps, $1 \mathrm{RM}$ y $\mathrm{VO}_{2}$ máx. Con estos resultados, concluyen que la oclusión vascular genera efectos similares, en cuanto a adaptaciones neuromusculares y cardiorespiratorias, que el entrenamiento concurrente en doce semanas de entrenamiento.

En el presente estudio no se encontró diferencia en la circunferencia del brazo después de cuatro semanas de entrenamiento de curl de bíceps de pie. Esto pudo deberse a que no que no se midió la hipertrofía por medio de resonancia magnética, solo se midió la circunferencia de brazo como un parámetro de hipertrofia, dejando de lado el porcentaje de grasa y músculo. Además, la mayoría de los estudios analizados encuentran estas diferencias en músculos del tren inferior, en conjunto con una ingesta adecuada de proteínas (Loenneke \& Pujol 2009; Sousa, et al., 2017; Vanwye, Weatherholt \& Mikesky, 2017). 


\section{CONCLUSIÓN}

El entrenamiento con oclusión vascular no genera un aumento en la circunferencia del brazo, sin embargo, mejora la resistencia muscular de la misma manera que el entrenamiento tradicional en cuatro semanas de entrenamiento. Cabe mencionar que el entrenamiento tradicional se realiza a una moderada intensidad (65\% 1RM) y el de oclusión vascular a baja intensidad $(20 \% 1 \mathrm{RM})$. Una recomendación para futuros estudios es aumentar la presión de la oclusión vascular a más del $5 \%$, es decir, limitar en mayor medida el flujo sanguíneo puede conseguir activar los mecanismos que benefician sobre este tipo de tratamientos, logrando una mayor ganancia en un menor tiempo. También, se recomienda hacer uso de resonancia magnética o ultrasonido directo, de esta manera, se obtienen datos precisos de oclusión vascular y dimensiones musculares para determinar el flujo sanguíneo y la hipertrofia.

Es importante involucrar a la población adulto mayor (mujeres y hombres) e interactuar con diferentes tipos de ejercicios (entrenamiento concurrente, entrenamientos interválicos de alta intensidad) en diferentes aparatos (cicloergómetro, remoergómetro, banda sinfín, etc) e intensidades de oclusión vascular.

NOTA: El presente estudio fue presentado en el 3er Simposio Internacional de Investigación Aplicada a la Actividad Física y Deporte y 2do Simposio Internacional de Tópicos Avanzados en Fisiología del Ejercicio, realizado en Tijuana, Baja California, México.

Agradecemos al Ph. D José Moncada Jiménez, de la Universidad de Costa Rica, por su colaboración en este proyecto.

\section{REFERENCIAS BIBLIOGRÁFICAS}

American College of Sports Medicine. (2009). American College of Sports Medicine position stand. Progression models in resistance training for healthy adults. Medicine \& Science Sports \& Exercise, 34(2), 364-380.

American College of Sports Medicine. (2005). Manual ACSM para la valoración y prescripción del ejercicio. Editorial Paidotribo.

Baechle, T.R., Earle, R.W., \& Wathen, D. (2000) Resistance training. In: Baechle, T.R. \& Earle, R.W., eds. Essentials of strength training and conditioning. 2nd ed. Champaign, IL: Human Kinetics, p. 395-425.

DeWeese, B. H., Hornsby, G., Stone, M., \& Stone, M. H. (2015). The training process: Planning for strength-power training in track and field. Part 1: Theorical aspects. Journal of Sport and Health Science, 4(4), 308-317.

Dankel, S., Jessee, M., Abe, T., \& Loenneke, J. (2016). The effects of blood flow restriction on upper-body musculature located distal and proximal to applied pressure. Sports Medicine, 46, 23-33.

Fahs, C., Loenneke, J., Thiebaud, R., Rossow, L., Kim, D., Abe, T., Beck, T., Feeback, D., Bemben, D., \& Bemben, M. (2014). Muscular adaptations to fatiguing exercise with and without blood flow restriction. Clinical Physiology and $\mathrm{Nu}$ clear Medicine, 35(3), 167-176.

Garber, C. E., Blissmer, B., Deschenes, M. R., Franklin, B. A., Lamonte, M. J., Lee, I.M., ... Swain, D. P. (2011). Quantity and Quality of Exercise for Developing and Maintaining Cardiorespiratory, Musculoskeletal, and Neuromotor Fitness in Apparently Healthy Adults. Medicine \& Science in Sports \& Exercise, 43(7), 1334-1359. https://doi.org/10.1249/ MSS.0b013e318213fefb 
George, J. D., Fisher, A., \& Vehrs, P. (2005). Test y pruebas físicas. Barcelona: Paidotribo.

Libardi, C. A., Chacon-Mikahil, M., Cavaglieri, C., Tricoli, V., Roschel, H., Vechin, F., Conceicao, M., \& Ugrinowitsch, C. (2014). Effect of concurrent training with blood flow restriction in the elderly. International Journal of Sports Medicine, 36, 395-399.

Lin, J., \& Chen, T. (2012). Diversity of strength training methods: A theoretical approach. Strength \& Conditioning Journal, 34(2), 42-49.

Lixandrão, M. E., Ugrinowitsch, C., Laurentino, G., Libardi, C. A., Aihara, A. Y., Cardoso, F. N., Tricoli, V., \& Roschel, H. (2015). Effects of exercise intensity and occlusion pressure after 12 weeks of resistance training with blood-flow restriction. European Journal of Applied Physiology, 115(12), 2471-2480.

Loenneke, J., \& Pujol, T. (2009). The Use of Occlusion Training to Produce Muscle Hypertrophy. Strength \& Conditioning Journal, 31(3), 77-84

Loenneke, J. P., Fahs, C. A., Thiebaud, R. S., Rossow, L., Abe, T., Ye, X., Kim, D., \& Bemben, M. (2012). The acute muscle swelling effects of blood flow restriction. Acta Physiologica Hungarica, 99(4), 400-410.

Loenneke, J. P., Fahs, C. A., Wilson, J. M., \& Bemben M. (2011). Blood flow restriction: the metabolite/volume threshold theory. Medical Hypotheses, 77(5), 748752.

Lowery, R., Joy, J., Loenneke, J., de Souza, E., Machado, M., Dudeck, J., \& Wilson, J. (2013). Practical blood flow restriction training increases muscle hypertrophy during a periodized resistance training programme. Clinical Physiology and Functional Imaging, 34(4), 317-321.
Nielsen, J. L., Aagaard, P., Bech, R. D., Nygaard, T., Hvid, L., Wernbom, M., Suetta, C., \& Frandsen, U. (2012). Proliferation of myogenic stem cells in human skeletal muscle in response to low-load resistance training with blood flow restriction. The Journal of Physiology, 590(17), 4351-4361.

Ogasawara, R., Loenneke, J. P., Thiebaud, R. S., \& Abe, T. (2013). Low-load bench press training to fatigue results in muscle hypertrophy similar to high-load bench press training. International Journal of Clinical Medicine, 4, 114-121.

Sainas, G., Milia, R., Palazzolo, G., Ibba, G., Marongiu, E., Roberto, S., Pinna, V., Ghiani, G., Tocco, F., \& Crisafulli, A. (2016). Mean blood pressure assessment during post-exercise: result from two different methods of calculation. Journal of Sports Science \& Medicine, 15(3), 424-433.

Sousa, J., Neto, G., Santos, H., Araújo, J., Silva, H., \& Cirilo-Sousa, M. (2017). Effects of strength training with blood flow restriction on torque, muscle activation and local muscular endurance in healthy subjects. Biology of Sport, 34(1), 83-90.

Takarada, Y., Sato, Y., \& Ishii, N. (2002). Effects of resistance exercise combined with vascular occlusion on muscle functions in athletes. European Journal of Applied Physiology, 86(4), 308-314.

Takarada, Y., Tsuruta, T., \& Ishii, N. (2004). Cooperative effects of exercise and occlusive stimuli on muscular function in low-intensity resistance exercise with moderate vascular occlusion. Japanese Journal of Physiology, 54(6), 585-592.

Takarada, Y., Takasawa, H., Sato, Y., Takebayashi, S., Tanaka, Y., \& Ishii, N. (2000). Effects of resistance exercise combined with moderate vascular occlusion on muscular function in humans. Journal of Applied Physiology, 88(6), 2097-2106. 
Vanwye, W., Weatherholt, A., \& Mikesky, A.

(2017). Blood flow restriction training: Implementation into clinical practice. International Journal of Exercise Science, 10(5), 649-654.

Vechin, F., Libardi, C., Conceicao, M., Damas, F., Lixandrao, M., Berton, R., Tricoli, V., Roschel, H., Cavaglieri, C., Chacón-Mikahil, M., \& Ugrinowitsch, C. (2015). Comparison between lowintensity resistance training with blood flow restriction and high-intensity resistance training on quadriceps muscle mass and strength in elderly. Journal of Strength and Conditioning Research, 29(4), 1071-1076.

Verkhoshansky, Y., \& Siff, M. (2011). Superentrenamiento. 2nd ed. Badalona: Paidotribo, p.22-23.

\section{Dirección para correspondencia}

Jorge Alberto Aburto-Corona

Maestría en Ciencias del Movimiento Humano

Universidad Autónoma de Baja California

Facultad de Deportes

Calzada Universidad \#14418 Parque Industrial Internacional Tijuana.

CP 22427, Tijuana B. C. México.

Contacto:

jorge.aburto@uabc.edu.mx

Fecha Recibido: 19-09-2018

Fecha Aceptado: 26-12-2018 